\title{
Third-party Reimbursement of Pharmacist-Led Cardiovascular and Diabetes Preventive Health Services for Workplace Health Initiatives: A Narrative Systematic Review
}

Brandon Tong, PharmD; Anita I. Kapanen, MSc, PhD; Jamie Yuen, BSc(Pharm), RPh, BCGP

Faculty of Pharmaceutical Sciences, The University of British Columbia, 2405 Wesbrook Mall, Vancouver, BC, Canada

\begin{abstract}
Objective: To summarize available literature describing third-party payer reimbursement models for pharmacist-led preventive health services as part of workplace health initiatives.

Methods: A combination of search terms related to pharmacists, preventive health, and third-party reimbursement were searched in MEDLINE, EMBASE, and PubMed. Included studies described community pharmacist-led cardiovascular and diabetes preventive health service to employees older than 18 years of age as part of a workplace health program with corresponding third-party reimbursement models. Programs that were reimbursed by government resources or studies lacking reimbursement model details were excluded. One reviewer performed level 1 screening and three reviewers analyzed included studies.

Results: The search criteria yielded 863 results. Sixteen articles were reviewed after level 1 screening and 13 were ineligible and excluded. Three studies with varying quality of reporting were included. Reimbursement models varied from $\$ 40$ USD for a 20 -minute visit to \$391 to \$552 USD total per patient with an average of 6 visits per patient.

Conclusion: There is a lack of quality literature describing third-party reimbursement models for pharmacist-led preventive health services, which hinders the ability to implement a standardized model. High quality studies evaluating the cost of reimbursing pharmacist-led cardiovascular preventive health services compared to the savings to the third-party payer should be performed to inform the standardization of payment models.
\end{abstract}

Keywords: cardiovascular; preventive health; reimbursement

\section{Introduction}

Pharmacists' role and scope of practice have expanded to include clinical services in the outpatient setting such as medication review programs, prescribing, point of care testing and immunizations. ${ }^{1,2,3}$ Offering these initiatives as part of outpatient pharmacy practice creates an opportunity to provide preventive health services. Preventive health care encompasses the prevention or occurrence of a disease or by providing health screening services, education, selfmanagement techniques or medication related interventions. ${ }^{4}$ Previously, pharmacists have offered preventive health services such as smoking cessation, cardiovascular risk reduction and immunization programs to improve public health. ${ }^{1,4}$ Additionally, pharmacist-led screening and coaching for diabetes, hypertension, and osteoporosis play a key role in disease identification, patient education, and referral of at risk patients. ${ }^{5}$ Preventive health programs initiated by workplace organizations or employers have demonstrated both clinical improvements and cost-savings in employee health outcomes, productivity and absenteeism. ${ }^{6,7,8,9}$

Corresponding author: Jamie Yuen, BSc (Pharm), RPh, BCGP

Faculty of Pharmaceutical Sciences

The University of British Columbia

2405 Wesbrook Mall, Vancouver, BC, Canada, V6T $1 Z 3$

Phone: 604-827-0704

Email: jamieh.yuen@ubc.ca
Although pharmacists have been shown to improve patient outcomes by providing medication management and preventive health services, a significant barrier for pharmacist uptake, practical implementation and maintenance of these programs is the lack of reimbursement by private, non-governmental, third-party payers. ${ }^{4,10}$ As third-party reimbursement models for pharmacist clinical services are in its infancy, pharmacists, other healthcare practitioners such as physicians and nurse practitioners, and third-party insurance providers need to be better informed of the utility and costsaving potential of pharmacist-led preventive health services. 4,10

\section{Objective}

The objective of this study is to summarize available literature describing third-party payer reimbursement models for pharmacist-led cardiovascular and diabetes preventive health services as part of workplace health initiatives.

\section{Methods}

This review follows the PRISMA guidelines for reporting systematic reviews. The protocol for the study was also registered on PROSPERO (CRD42018104986).

\section{Search Strategy}

MEDLINE, EMBASE, and PubMed were searched from 1946 to November 2019. The search method comprised of three main topics: pharmacists, preventive health services, and third-party reimbursement. A combination of various $\mathrm{MeSH}$ terms and keywords were used and tailored for each database. The search 
terms used for MEDLINE include pharmacist* OR exp Pharmacists/[MeSH] OR pharmacy.mp AND exp Health Education/[MeSH] OR exp Preventative Health Services/[MeSH] OR exp Preventive Health Services/[MeSH] OR exp Community Pharmacy Services/[MeSH] OR exp Public Health/[MeSH] AND exp Insurance, Health, Reimbursement/[MeSH] OR reimbursement.mp OR thirdparty.mp. Our search was limited to peer-reviewed North American studies written in the English language to improve the generalizability of our findings to the North American practice setting.

\section{Study selection}

The original intent was to include studies evaluating preventive health services for chronic diseases, however, based on the lack of information on third-party private reimbursement, the decision was made to focus on cardiovascular and diabetes populations. Included studies described a community pharmacist-led cardiovascular and diabetes preventive health service to employees older than 18 years of age as part of a workplace health program. Details of the third-party reimbursement model for the pharmacist services were required to be included. Studies evaluating pharmacist services that were reimbursed by the government or paid for by the patient were excluded.

One reviewer was responsible for screening titles from the search results for review (level 1). Reviewer one and two additional independent reviewers, independently appraised the studies using the Joanna Briggs Institute appraisal tools. ${ }^{11}$ After discussion by all three reviewers, a final decision was made regarding study inclusion.

Due to the low number of results, all studies meeting the inclusion criteria were included in the review and a formal risk of bias assessment was not performed.

\section{Results}

The initial search yielded 863 results. Following the removal of duplicate results and level 1 screening, 16 full-text articles were then assessed for eligibility and 13 were excluded. Although many published studies reported third-party reimbursement for pharmacist interventions, they lacked a detailed description of the reimbursement model. Unfortunately, attempts at contacting the authors for clarification were unsuccessful and as a result, they were excluded from the review. In total, 3 articles were identified that fit the inclusion criteria (Figure 1).

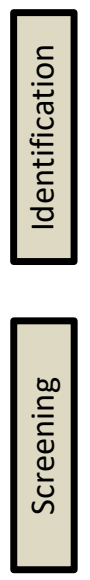

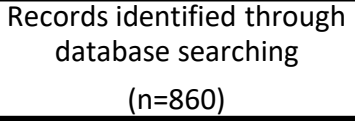

Additional records identified through other sources

$(n=3)$

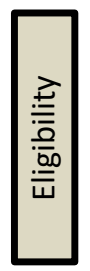

Records screened

$(n=602)$ removed

$(n=602)$

Full-text articles assessed for eligibility $(n=16)$

Studies included in qualitative synthesis $(n=3)$
Records excluded

$(n=573)$
Full-text articles excluded, with reasons $(n=13)$

Figure 1. Study Selection Flow Diagram 
In the Asheville Project, a quasi-experimental longitudinal prepost study, a community pharmacy diabetes care program was provided for employees of the City of Asheville and Mission-St. Joseph's Health System. ${ }^{12}$ One-hundred eighty seven participants were enrolled and attended consultations with a pharmacist trained in diabetes care who conducted physical assessments and provided diabetes education and treatment monitoring goals on an ongoing basis. This program was provided at no cost to participants and was fully covered by the self-insured employers and their respective third-party providers. The fee for service model averaged \$40 USD for a 20minute visit. Patients demonstrated statistically significant reductions in mean hemoglobin A1c concentrations and at all follow-ups (6 month intervals following baseline), over $50 \%$ patients had improved hemoglobin A1c values. Additionally, 24.3\% more patients had optimal hemoglobin A1c values (hemoglobin $\mathrm{A} 1 \mathrm{c}<7 \%$ ) at the first follow-up, and increases of $27.2 \%$ and $18.2 \%$ at the second and third follow-ups respectively. After analyzing the study's economic data from 164 patients, third-party payers experienced a reduction in annual expenses by $\$ 1622$ USD to $\$ 3356$ USD per patient. The City of Asheville also reported a mean decrease in sick days by 4.1 to 6.6 days per participant per year from a baseline of 12.6 days. The estimated value of increased productivity was $\$ 18,000$ USD a year.

The Diabetes Ten City Challenge was a quasi-experimental observational pre-post study. ${ }^{13}$ It included 573 diabetic employees from 10 geographic locations, who received coaching from a pharmacist to achieve their clinical and selfmanagement goals. The various employers of the study were self-insured and worked with their respective third-party insurance administrators to invest in incentives for both employees and pharmacist providers. For pharmacists, this included reimbursement for their services. Over the span of approximately a year, 573 participants received a mean of 6 patient-pharmacist visits, with a mean of 51 minutes in duration for each visit. It was reported that the overall cost to the employer for pharmacist-patient visits was \$224,043 USD and on average, pharmacists received an estimated \$391 USD per patient in total with an average of 6 visits per participant. Over the course of 14.8 months, patients showed modest yet significant decreases in key clinical measures of hemoglobin A1c, LDL cholesterol, blood pressure, and BMI. Based on the projected medical costs from the economic analysis of the study's population, total cost-savings during the first year of program implementation were estimated at $\$ 339,875$ USD for patients and $\$ 278,512$ USD for employers. Additionally, there were increases to the number of patients who received influenza vaccinations (from $32 \%$ to $65 \%$ ), eye examinations (from $57 \%$ to $81 \%$ ) and foot examinations (from 34 to $74 \%$ ).

The Cincinnati Pharmacy Coaching Program for Diabetes and Hypertension was a quasi-experimental pre-post longitudinal study. ${ }^{14}$ Employees with diabetes or hypertension were invited to the program by employers and health plan communicators. Those enrolled regularly followed up with community-based pharmacists who provided education and monitoring. The study consisted of 607 intervention participants separated into a diabetes or a hypertension coaching program cohort and 557 propensity score-matched control participants. Pharmacist coaching program costs for the hypertension and diabetes cohorts were \$493 USD (+/-256) and \$552 USD (+/-350) per participant respectively with each participant averaging 6 visits. The direct costs to study participants were waived and paid for by the employer. Blood pressure and LDL cholesterol values improved significantly from baseline for both groups and within the diabetes cohort, mean hemoglobin A1c levels improved significantly (from $7.9 \%$ to $7.1 \%$ ). After a review of economic data, the program resulted in positive cost trends. Total hypertension-related and all-cause costs were lower, but not statistically significant. The intervention led to a significant decrease in hypertension-related ER visit costs (39.2\% vs $16 \%$ ). For the diabetes program, total medical costs increased $11 \%$ for the intervention group and approximately $300 \%$ for the control cohort $(p<0.05)$. In addition, diabetes-related ER visit costs decreased by $89 \%$ in the intervention group and increased $96 \%$ for the control group $(p<0.05)$.

\section{Discussion}

Pharmacists have demonstrated value for improving patient outcomes and providing cost-savings through a preventive health approach. ${ }^{4}$ However, currently third-party payers do not routinely provide reimbursement for pharmacist clinical services and most of the current literature supporting thirdparty reimbursement for pharmacist clinical services unfortunately do not provide a comprehensive description of the payment model. ${ }^{2,15,16}$ A major barrier to third-party reimbursement of pharmacist-led preventive health services can be attributed to the fact that pharmacists are currently not recognized providers by the Centers for Medicare and Medicaid Services (CMS). ${ }^{17}$ This review provides a summary of existing literature describing pharmacist-led preventive health services as part of a workplace health initiative with details regarding a third-party payment model.

The included studies had a range in reimbursement models from \$40 USD for a 20-minute visit to \$391 to \$552 USD total per patient with an average of 6 visits per patient. ${ }^{12,13,14}$ Guidance on how to approach establishing a third-party payer reimbursement model is detailed in available literature. ${ }^{15}$ Proposed models may include reimbursement by individual patient, type of visit (initial or follow up), service provided, or time spent with the patient. ${ }^{15}$ One approach to third-party billing by individual pharmacists is to become approved by the insurance company as a health care service provider for their clients in a process known as credentialing. Green Shield Canada has launched an initiative that remunerates pharmacist coaching services for cardiovascular health interventions after completion of their training program. ${ }^{18}$ Patients covered under 
Green Shield extended health benefits, who are under the age of 65 and taking a qualifying cardiovascular drug regimen, are eligible for this coaching service. Pharmacists are compensated $\$ 60$ CAD for an initial visit and \$20 CAD for each follow-up visit (up to 3 within 1 year of the initial visit). ${ }^{19}$ Alternatively, pharmacists may receive compensation through value-based insurance designs (V-BID) developed by health insurance companies and utilized by employers. Plan providers encourage the usage of preventive health services for their members and pharmacists are compensated for patient visits. ${ }^{15}$ In our review, the Asheville Project, the Diabetes Ten City Challenge, and the Cincinatti Pharmacy Coaching program all utilized a form of value-based insurance design for pharmacist reimbursement. There are also compensation models used to reimburse pharmacist clinical services through government healthcare plans that pharmacists may consider when starting value-based initiatives. ${ }^{20}$ The average remuneration value for medication review services not specific to preventive health was $\$ 68.86$ CAD for an initial visit and \$23.37 CAD for follow ups. ${ }^{10}$ Thirdparty payers may consider adapting similar payment models based on cost outcomes from government initiatives such as medication therapy management programs. ${ }^{21}$ In the context of preventive health services, cost of point of care testing equipment and supplies should also be taken into consideration in addition to pharmacist services.

Studies of high quality design and longer duration are required to further gain insight into third-party reimbursement models for pharmacist-led preventive health services. Clinical outcomes established by investigators may impact the potential for cost benefits to manifest as certain surrogate outcomes and resulting impact on health related costs may not be immediately apparent. As select clinical outcomes have been demonstrated through pharmacist-led interventions, additional focus should be placed on the cost benefits for third-party insurers. ${ }^{4}$ Future evaluations should consider the goal of establishing pharmacist-led preventive health services as a permanent service covered by third-party insurance as implemented in the Asheville Project. ${ }^{12}$

\section{Limitations}

The review was limited by the paucity of quality literature describing third-party reimbursement of pharmacist-led preventive health programs. A major limitation identified in this review was the lack of detail in reporting of third-party reimbursement models. Available literature primarily focused on the reporting of clinical outcomes rather than the economic aspect of their evaluation. In addition, there was significant heterogeneity of the study designs and data, limiting the generalizability and ability to directly compare the results. Studies were generally short in duration, which may have impacted potential cost benefit of pharmacist-led preventive health services.

\section{Conclusion}

The studies included in this review were able to demonstrate clinical and cost-savings benefits of pharmacist preventive health intervention and justify the need for a proper reimbursement model to compensate pharmacists for their professional services. In our review, all studies utilized a valuebased insurance design to successfully receive reimbursement for pharmacist services. Future areas to explore include high quality studies evaluating the cost of reimbursing pharmacistled cardiovascular and diabetes preventive health services compared to the savings to the third-party payer to inform the standardization of payment models.

\section{References}

1. Tsuyuki RT, Johnson JA, Teo KK, et al. A randomized trial of the effect of community pharmacist intervention on cholesterol risk management: the study of cardiovascular risk Intervention by pharmacists (SCRIP). Arch Intern Med. 2002 May 27 ; 162(10): 1149-55.

2. Mehta $\mathrm{BH}$, Rodis JL, Nahata $\mathrm{MC}$, et al. Advancing patient care through innovative practice: the clinical partners program. Am J Health Syst Pharm. 2005 Dec 1; 62(23): 2501-7.

3. Canadian Pharmacists Association. Pharmacists' scope of practice in Canada. Available from: https://www.pharmacists.ca/cpha-ca/assets/File/cpha-on-theissues/Scope\%20of\%20Practice\%20in\%20Canada April2019.pdf (accessed 202001 31)

4. San-Juan-Rodriguez A, Newman TV, Hernandez I, et al. Impact of community pharmacist-provided preventive services on clinical, utilization, and economic outcomes: an umbrella review. Prev Med. 2018 Oct; 115: 145-155.

5. Jean-Venable "Kelly" Goode, Swiger K, Bluml B. Regional Osteoporosis Screening, Referral, and Monitoring Program in Community Pharmacies: Findings from Project ImPACT: Osteoporosis. Journal of the American Pharmacists Association. 2004;44(2):152-160.

6. Groeneveld I, Proper K, van der Beek A, et al. Lifestyle-focused interventions at the workplace to reduce the risk of cardiovascular disease - a systematic review. Scand J Work Environ Health. 2010 May; 36(3): 202-15.

7. Cancelliere C, Cassidy J, Ammendolia C. Are workplace health promotion programs effective at improving presenteeism in workers? A systematic review and best evidence synthesis of the literature. BMC Public Health. 2011 May 26; 11:395.

8. 8 Gobis B, Kapanen Al, Reardon J, et al. Cardiovascular risk reduction in the workplace with CAMMPUS (Cardiovascular Assessment and Medication Management by Pharmacists at the UBC Site). Ann Pharmacother. 2019 Jun; 53(6): 574-580. 
9. Zhang W, Li KH, Gobis B, et al. Work productivity losses and associated risk factors among university employees in the CAMMPUS wellness program. J Occup Environ Med. 2020 Jan; 62(1): 25-29.

10. Houle SK, Grindrod KA, Chatterley T, et al. Paying pharmacists for patient care: a systematic review of remunerated pharmacy clinical care services. Can Pharm J (Ott). 2014 Jul; 147(4): 209-32.

11. Joannabriggs critical appraisal tools for use in JBI systematic reviews. Available from: https://joannabriggs.org/sites/default/files/2020-08/Checklist for_Case Reports.pdf. (accessed 20201214 )

12. Cranor CW, Bunting BA, Christensen DB. The Asheville project: long-term clinical and economic outcomes of a community pharmacy diabetes care program. J Am Pharm Assoc (Wash). 2003 Mar-Apr; 43(2): 173-84.

13. Fera T, Bluml BM, Ellis WM. Diabetes ten city challenge: final economic and clinical results. J Am Pharm Assoc (2003). 2009 May-Jun; 49(3): 383-91.

14. 14 Wertz D, Hou L, DeVries A, et al. Clinical and economic outcomes of the Cincinnati pharmacy coaching program for diabetes and hypertension. Manag Care. 2012 Mar; 21(3): 44-54.

15. Snella KA, Trewyn RR, Hansen LB, et al. Pharmacist compensation for cognitive services: focus on the physician office and community pharmacy. Pharmacotherapy. 2004 Mar; 24(3): 372-88.

16. Lloyd KB, Evans RL. Reimbursement model for pharmacist-directed medication therapy management. J Am Pharm Assoc (2003). 2012 Mar-Apr; 52(2): 161-9.

17. Provider status: What pharmacists need to know now. Available from: https://www.pharmacist.com/provider-status-whatpharmacists-need-know-now. (accessed 202012 16)

18. Provider Connect. Pharmacist health coaching - cardiovascular program information. Available from: https://www.providerconnect.ca/HealthCoaching/ProgramInformation.aspx (accessed 20200131 )

19. Provider Connect. Pharmacist Health Coaching. Cardiovascular Program. Available from: https://www.providerconnect.ca/wwwroot/ProviderForms/HealthCoaching/GSC\%20Cardiovascular\%20Program Program \%20Guide vf.pdf. (accessed 202012 15)

20. Scott MA, Hitch WJ, Lugo AM. Billing for pharmacists' cognitive services in physicians' offices: multiple methods of reimbursement. J Am Pharm Assoc (2003). 2012 Mar-Apr; 52(2): 175-80.

21. Houle S, Grindrod K, Chatterley T, Tsuyuki R. Paying pharmacists for patient care. Canadian Pharmacists Journal / Revue des Pharmaciens du Canada. 2014;147(4):209-232. 